\title{
POST PERCUTANEOUS NEPHROLITHOTOMY (PCNL) RENAL ANGIOEMBOLIZATION- EXPERIENCE AT OUR CENTRE (IGIMS)
}

\author{
Ahsan Ahmad', Gaurav², Kumar Gaurav Mishra ${ }^{3}$, Vishrut Bharti ${ }^{4}$
}

${ }^{1}$ Associate Professor, Department of Urology, IGIMS, Patna, Bihar, India.

${ }^{2} M c h 2^{\text {nd }}$ Year, Department of Urology, IGIMS, Patna, Bihar, India.

${ }^{3}$ Mch $3^{\text {rd }}$ Year, Department of Urology, IGIMS, Patna, Bihar, India.

${ }^{4}$ Mch 1 st Year, Department of Urology, IGIMS, Patna, Bihar, India.

\begin{tabular}{l}
\hline ABSTRACT \\
PACKGROUND \\
multiple calculus with minimal post-operative complication rate. Our study evaluates the management of postoperative hematuria \\
and searches for pre-operative predictors of post PCNL bleeding.
\end{tabular}

\section{METHODS}

This descriptive study with patients who had PCNL done for renal calculus were retrospectively evaluated between July 2016 to June 2017. They were segregated into two groups; one group was having significant hematuria and other group was having minimal hematuria after applying inclusion and exclusion criteria. We compared different patient related factors like age \& sex of patient, presence of hypertension, diabetes, renal failure, UTI, past history of renal surgeries, and side of surgery with indication of surgery for identifying patient having significant post-operative hematuria.

\section{RESULTS}

Out of 250 patients, only 5 patients required renal angiography and 4 patients required angioembolization for significant post-op hematuria.

\section{CONCLUSIONS}

Post PCNL angioembolization of the injured vessel is an effective, minimally invasive and relatively easy procedure in experienced centers, with high success rate and immediate benefits, thus saving the patient from the morbidity that results from severe renal haemorrhage after PCNL.

HOW TO CITE THIS ARTICLE: Ahmad A, Gaurav, Mishra KG, et al. Post percutaneous nephrolithotomy (PCNL) renal angioembolization- experience at our centre (IGIMS). J. Evolution Med. Dent. Sci. 2019;8(16):1255-1257, DOI: $10.14260 /$ jemds/2019/279

\section{BACKGROUND}

Percutaneous access to the kidney was first described in 1865 by Thomas Hillier who repeatedly drained a 4-year-old boy's kidney, which he thought at that time to be congenitally obstructed. Percutaneous nephrolithotomy (PCNL) for stone disease was first described in the 1970s by Fernstrom and Johannson.[1] Presently, the clinical practice guideline report for the management of staghorn calculi, by the AUA and European Urological Association, recommend that percutaneous treatment of staghorn calculi should be firstline treatment for most patients. [2]

Percutaneous nephrolithotomy (PCNL) is an effective modality of management of large renal calculus in terms of early recovery and stone clearance rates when compared to open surgery and ESWL.[3] Kidney has got high vascular network which lies in the vicinity of PCS. During inadvertent access to the PCS there may be injury to these vessels which may cause post PCNL hematuria.[4]

'Financial or Other Competing Interest': None.

Submission 05-03-2019, Peer Review 09-04-2019,

Acceptance 15-04-2019, Published 22-04-2019.

Corresponding Author:

Kumar Gaurav Mishra,

Flat No. 31,

New M. D. H,

IGIMS Campus, Patna,

Bihar, India.

E-mail: gauravmishra.pmch@gmail.com

DOI: $10.14260 /$ jemds $/ 2019 / 279$

\section{(c) $(1)$}

This is One of the fearsome postoperative complication of PCNL. [5] However conservative measures are adequate to control bleeding in most cases. Angioembolization and further procedures are required in $1 \%$ of patients to control persistent bleeding.

\section{METHODS}

This descriptive study with patients who had PCNL done for renal calculus were retrospectively evaluated between July 2016 to June 2017. They were segregated into two groups, one group was having significant hematuria and other group was having minimal hematuria after applying inclusion and exclusion criteria. A number of 24 haemorrhagic complications were observed, but only 5 patients $(2 \%)$ had significant bleeding that required angiography and only 4 required embolization for bleeding control. Hemogram, coagulation profile, serum electrolytes, glucose, serum creatinine levels, urinalysis and urine culture, liver function tests, ultrasonography and intravenous urography and CT urography (In selected patients) were performed before the patients underwent PCNL. Our operative technique involved percutaneous puncture under multidirectional C-arm fluoroscopic guidance, dilation of the nephrostomy tract up to 28F, and use of an Amplatz sheath. Smaller stones were removed with the help of forceps, larger ones being broken with pneumatic lithotripsy before extraction. The nephrostomy tube was retained only in cases with a stone burden of $3 \mathrm{~cm}$ or more, multiple access tract, significant residual stones, perforation, bleeding, and requirement for a 
secondary percutaneous procedure. In the rest of the patients we have used 'tubeless' PCNL, in which the nephrostomy tube was replaced with internal drainage provided by a double-J stent or a ureteral catheter.

After informed consent was obtained and digital subtraction angiography confirmed the diagnosis of pseudoaneurysm (PA) or arteriovenous fistula (AVF), percutaneous embolization was performed by our interventional cardiologist. For the DSA procedure, the left brachial or right main femoral artery was punctured under local anaesthesia using Seldinger technique and a $6 \mathrm{~F}$ vascular sheath was inserted as an introducer (Cordis, Johnson \& Johnson, Miami, USA). Over a 0.035-inch diameter guidewire (Terumo, Osaka, Japan), an abdominal aortography was obtained with a $5 \mathrm{~F}$ Cobra catheter (Cordis, Johnson \& Johnson, Miami, USA) by injecting a 8-10 ml contrast media, which shows the main or accessory renal arteries on either side. Thereafter, a selective renal DSA was performed by advancing the catheter into the injured branch of the renal artery feeding the lesion. Rapid filming sequences and careful examination of all phases of the arteriogram are necessary to assess the site and feeding pedicle, flow pattern and venous drainage of the vascular lesion.

\section{RESULTS}

Of the 250 PCNL procedures, 5 patients (2.0\%), 4 males and 1 female, aged 28-64 years (mean, 46), required super selective renal angiography due to severe/persistent hematuria from the right (3 cases) or left kidney (2 cases). The diagnosis present at admission was of unilateral renal stone in all 5 patients. Table 1 lists patient's demographic profile and surgical procedure characteristics in cases requiring angiographic imaging.

As the first imaging modality, USG was used in five patients after then they went DSA. In The indications for renal DSA were: severe hematuria with hemodynamic instability associated with important decrease of the haemoglobin and haematocrit values during the first 24 hours after surgery (1 patient); frank renal haemorrhage necessitating blood transfusion in the early postoperative period, 3 to 14 days after PCNL (3 patients).In one patient DSA was indicated on post-operative day 23. The severity of the hematuria after PCNL is influenced by many factors, including mean stone size and mean operative time and is correlated with duration of hospitalization and mean haemoglobin drop.

Renal angiography revealed pseudoaneurysm in 2 patients [Fig. 1 \& 2], arteriovenous fistula in 2 and 1 patient showed normal angiography. Significant risk factors on univariate analysis for severe hematuria requiring super selective angiography were multiple/staghorn calculi, upper calyx puncture and history of pyelonephritis. The severity of the hematuria after PCNL is influenced by many factors, including mean stone size and mean operative time and is correlated with duration of hospitalization and mean haemoglobin drop. Super selective angioembolization was done in 4 patients while one patient was managed conservatively. After angioembolization all patients were asymptomatic and on regular follow up they have no any complain.

\begin{tabular}{|c|c|c|c|}
\hline & Character & Number & $\mathbf{\%}$ \\
\hline \multirow{2}{*}{ Mean Age 28-64 } & Male & 4 & 80 \\
& Female & 1 & 20 \\
\hline \multirow{2}{*}{ Side } & Right & 4 & 80 \\
& Left & 1 & 20 \\
\hline \multirow{3}{*}{ Stone Burden } & Single & 1 & 20 \\
& Multiple & 1 & 40 \\
& Staghorn & 3 & 40 \\
\hline \multirow{2}{*}{ Number of Tracts } & Single & 3 & 60 \\
& Multiple & 2 & 40 \\
\hline \multirow{2}{*}{ History of Urinary } & None & 4 & \multirow{2}{*}{ Tract Infection } \\
& Uncomplicated & 1 & 20 \\
& Pyelonephritis & 0 & \\
\hline Concurrent Medical & Diabetes Mellitus & 1 & 20 \\
Illnesses & Hypertension & 1 & 20 \\
\hline
\end{tabular}

Table 1. Patient and Surgical Procedure Characteristics of 5 Cases Requiring Angiography

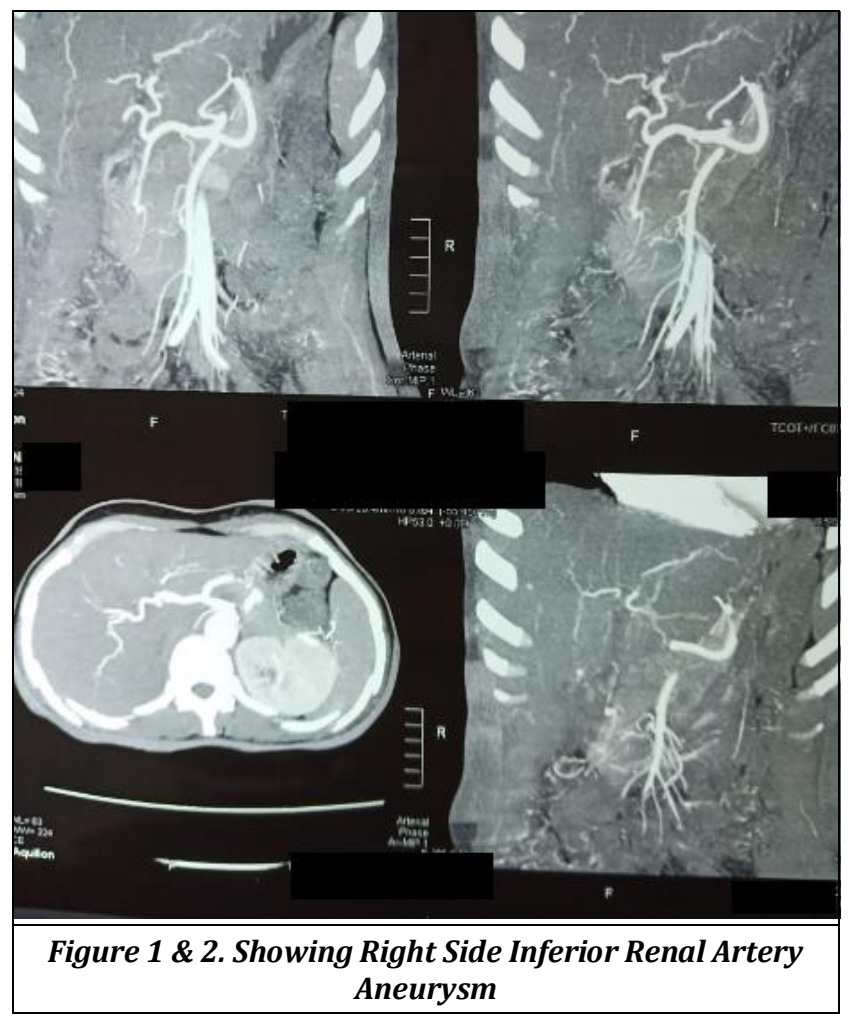

\section{DISCUSSION}

One of the dangerous post-operative complications of PCNL is bleeding which may require blood transfusion in 1 to $11 \%$ cases.[6] This may be due to trauma to intrarenal arterio venous network.(7) Profuse haemorrhage may take place when getting access to desired stone bearing calyces intraoperatively or can manifest postoperatively.(4) pseudoaneurysm of renal arteries are caused by trauma during access or intraoperative manipulation. This may result in injury to renal artery or parenchyma. The blood from traumatised artery may percolate into low pressure vein causing pseudoaneurysm.(8) We had 4 patients out of 250 patients who had significant bleeding within two weeks after PCNL and one patient out of 250 who had delayed bleeding after 41 days of procedure. However there have been reports of bleeding occurring as late as after 13 to 14 weeks after PCNL. (9) 
In the initial management of massive bleeding we clamp nephrostomy tube for 3 to 7 hours and apply tight bandage over tract site.(6) In case of gross haematuria with clot urinary retention fluid resuscitation with blood transfusion should be given.(10)

If these management fail to resolve haematuria, then renal angiography is done to locate site of bleeding. Renal angiography done in our study showed arteriovenous fistula in 2 patients, pseudoaneurysm in 2 patients and normal angiographic finding in one patient. Super selective angioembolization is done to get control of bleeding aneurysm or arteriovenous fistula to avoid large area of renal parenchymal loss of blood supply leading to infarction of renal tissue as may happen in nonselective embolization. In our study success rate of super selective embolization 80 percent is comparable to other centre success rate of 72-95 percent. $(4,5,11,12,10,6)$

Bleeding complications are more common in diabetic patients, however high blood pressure and renal failure have also been identified as predictors of increased bleeding in other studies. In diabetic patients there may be atherosclerosis of renal arterioles causing increase risk of bleeding.(3)

Stoller et. al(13) did not find a relationship of postoperative bleeding with patient having prior surgery while Neeto et al had opposite finding in their studies.(14) Kukerja et al(15) found significantly less bleeding in previously operated patients. In our study we couldn't attribute prior surgical intervention as risk factor for postoperative bleeding. However, incidence of significant bleeding requiring blood transfusion(4-5) was more in staghorn calculi than solitary renal calculi as suggested by Krukeja et. al(15) and Srivastava et al(12) in their studies.

\section{CONCLUSIONS}

The gold standard treatment of large renal stone is per cutaneous nephrolithotomy (PCNL). However, post PCNL hematuria is sometimes severe enough to cause fatal complication resulting in death. Proper understanding of renal vascular anatomy and choosing proper site of puncture is essential in avoiding these complications. Identifying preoperative risk factor for increased chances of bleeding is of great importance in reducing postoperative bleeding. In our study, we identified preoperative risk factors like, urine culture, large volume stone disease, and diabetes as major risk factors for massive hematuria. However, in our experience, significant bleeding due to vascular injuries after PCNL could be managed conservatively with selective renal artery embolization. The high success rate of selective embolization with minimal complication was the reason for choosing this procedure in our study.

\section{REFERENCES}

[1] Fernstrom I, Johansson B. Percutaneous pyelolithotomy. A new extraction technique. Scand J Urol Nephrol 1976;10(3):257-9.

[2] Preminger GM, Assimos DG, Lingeman JE, et al. Chapter 1: AUA guideline on management of staghorn calculi: diagnosis and treatment recommendations. J Urol 2005;173(6):1991-2000.

[3] Pardalidis NP, Smith AD. Complications of percutaneous renal procedures. In: Controversies in Endourology. Philadelphia: WB Saunders 1995: p. 179.

[4] Kessaris DN, Bellman GC, Pardalidis NP, et al. Management of hemorrhage after percutaneous renal surgery. J Urol 1995;153(3 Pt 1):604-8.

[5] Gupta M, Bellman GC, Smith AD. Massive hemorrhage from renal vein injury during percutaneous renal surgery: endourological management. J Urol 1997;157(3):795-7.

[6] Huang WH, Jiann BP. Risk factors of massive bleeding after percutaneous nephrolithotomy and its management. JTUA 2003;14:65-71.

[7] Motola JA, Smith AD. Percutaneous techniques. Chap 9. In: Smith JA Jr, edr. Hightech urology: technical innovations and their clinical applications. Philadelphia: WB Saunders Co, 1992: p. 208-23.

[8] Cope C, Zeit RM. Pseudoaneurysms after nephrostomy. Am J Roentgen 1982;139(2):255-61.

[9] Gavant ML, Gold RE, Church JC. Delayed rupture of renal pseudoaneurysm: complication of percutaneous nephrostomy. Am J Roentgenol 1982;138(5):948-9.

[10] El-Nahas AR, Shokeir AA, El-Assmy AM, et al. Postpercutaneous nephrolithotomy extensive hemorrhage: a study of risk factors. J Urol 2007;177(2):576-9.

[11] Martin X, Murat FJ, Feitosa LC, et al. Severe bleeding after nephrolithotomy: results of hyperselective embolization. Eur Urol 2000;37(2):136-9.

[12] Srivastava A, Singh KJ, Suri A, et al. Vascular complications after percutaneous nephrolithotomy: are there any predictive factors? Urology 2005;66(1):38-40.

[13] Stoller ML, Wolf JS Jr, St Lezin MA. Estimated blood loss and transfusion rates associated with percutaneous nephrolithotomy. J Urol 1994;152(6 Pt 1):1977-81.

[14] Netto RN Jr, Lemos GC, Palma PC, et al. Staghorn calculi: percutaneous versus anatrophic nephrolithotomy. Eur Urol 1988;15(1-2):9-12.

[15] Kukreja R, Desai M, Patel S, et al. Factors affecting bloodloss during percutaneous nephrolithotomy: prospective study. J Endourol 2004;18(8):715-22. 